\title{
MULHERES NA ENGENHARIA: CAPACITAÇÃO EM ENERGIA SOLAR PARA MENINAS.
}

\author{
Alexandre M. Farias - alexandre.farias@ pesqueira.ifpe.edu.br \\ Manoel H. O. Pedrosa Filho - manoel@ pesqueira.ifpe.edu.br \\ Instituto Federal de Educação, Ciência e Tecnologia da Pernambuco, Campus Pesqueira. \\ BR 232, km 208, Prado. \\ CEP: 55200-000 - Pesqueira - PE
}

\begin{abstract}
Resumo: Com a publicação das Resolução Normativa 482/12 e 687/15 pela ANEEL, A geração de energia solar fotovoltaicas no Brasil começou a atingir um crescimento exponencial, proporcionando o surgimento de demandas de profissionais para atuarem em projetos e instalações de sistemas. A partir deste momento, diversos cursos rápidos começaram a ser ofertados com o objetivo de sanar esta deficiência. Porém, observava-se que estas capacitações não eram suficientes para qualificar um profissional nesta área e a partir de então, iniciou-se uma discussão entre as principais instituições de ensino tecnológico para a criação um itinerário formativo que permitisse capacitar estes profissionais de uma forma mais adequada. Este trabalho apresenta a metodologia e os resultados de um modelo de capacitação realizado especificamente para um grupo de alunas e professoras do Instituto Federal de Pernambuco em projetos e instalação de sistemas fotovoltaicos, com o objetivo de motiva-las a desbravar áreas que historicamente só os homens faziam. Os resultados serão usados também para motivar novas alunas a atuar nessa área. Este trabalho também descreverá o desenvolvimento de conteúdos abordados para a formação das alunas para atender às necessidades do mercado, como conceitos, técnicas $e$ materiais básicos de energia solar fotovoltaica para instalações em telhados. As principais práticas realizadas também são descritas, como a montagem dos componentes do conector MC4, da String Box e os procedimentos de instalação dos módulos fotovoltaicos no telhado (segurança e materiais).
\end{abstract}

Palavras-chave: Educação em Sistemas Fotovoltaicos. Mulheres na Engenharia. Sistema Solar Conectado à Rede

\section{INTRODUÇÃO}

O mercado de energia solar fotovoltaica no Brasil começou a se expandir a partir da regulamentação do setor pela Agência Nacional de Energia Elétrica (ANEEL) em 2012, a partir da publicação da Resolução Normativa 482/12 e em seguida, em 2015, sua atualização com a Resolução Normativa 687/15. Desde então, o número de sistemas fotovoltaicos vem aumentando exponencialmente. Entretanto, a formação de mão de obra qualificada para realizar as instalações dos sistemas não acompanhou a demanda crescente do setor. Além de capacitação profissional prática mais específica, também são necessários conhecimentos acerca do dimensionamento, proposição e gerenciamento de projetos, comissionamento, operação e manutenção, dentre outros detalhes técnicos. Dessa maneira, fez-se necessário elaborar uma iniciativa a nível nacional para motivar a criação de cursos de formação 
profissional na área (PEDROSA FILHO et al., 2018). Em geral a presença feminina nessas capacitações e na área das engenharias é baixa. No portal do Instituto Nacional de Estudos e Pesquisas Educacionais Anísio Teixeira (INEP) é possível obter alguns dados que comprovam a baixa participação feminina. Segundo o Censo da Educação Superior 2016, haviam 291.463 mulheres fazendo graduação em engenharia no Brasil. Esse número correspondia na época a $28,3 \%$ dos estudantes que optam por esse curso superior no país. Fazendo um balanço mais próximo, o número de alunas matriculadas no curso de Bacharelado em Engenharia Elétrica no campus Pesqueira nunca passou de 25\%, comprovando os baixos percentuais observados nos dados do INEP. Isso posto, a proposta principal desta capacitação foi mostrar que as mulheres podem exercer suas atividades em qualquer área da profissão. Nesse contexto, o Instituto Federal de Pernambuco, Campus Pesqueira, ofertou um curso básico de projetista e instaladora de sistemas fotovoltaicos focado na formação do publico feminino a fim de que estas jovens venham a contribuir para a sociedade executando projetos na região.

\subsection{O crescimento da energia solar fotovoltaica no país e as formações implementadas}

O mercado brasileiro se apresenta aquecido com essas taxas de crescimento e tende a manter esses números nos próximos anos. De acordo com os dados, obtidos no site da ANEEL, o percentual de potência instalada de mini e microgeradores solares fotovoltaicos comparados com o instalado no ano anterior foi de 455\% (2016), 137\% (2017), 211\% (2018) e $251 \%$ (2019), totalizando 1985,7 MWp de potência instalada. Já o número de instalações teve um acréscimo anual com relação ao ano anterior de 355\% (2016), 118\% (2017), 175\% (2018) e 214\% (2019), representando um total de 214.984 unidades consumidoras de energia solar fotovoltaica.

Há uma falta de mão-de-obra qualificada devido a essa área ser relativamente nova no Brasil e apresentar um crescimento rápido que não pode ser seguido pela capacidade de treinar pessoas para atuar nela. Segundo Mocelin (MOCELIN, 2014), a consequência da execução do projeto por pessoas não qualificadas podem ocasionar em más instalações e consequentemente provocar uma influência negativa na reputação da tecnologia, desacreditado a população.

Alguns problemas já foram verificados em algumas instalações no Brasil, como mau funcionamento do sistema: incêndio em caixas de junção (Figura 1a), incêndio em módulos fotovoltaicos (Figura 1b) e estrutura metálica mal avaliada (Figura 1c) que não suportavam a carga acima dele.

Iniciativas louváveis, ainda que pontuadas e isoladas, em algumas instituições brasileiras, podem ser observadas para suprir a falta de formação de mão de obra nessa área. Blasques (BLASQUES, 2016), Neto et al (NETO, 2016) e Diniz et al (DINIZ, 2016), por exemplo, apresentaram suas propostas de cursos nessa área. Existem cursos lato senso em diversas universidades no Brasil, destacando-se, por exemplo, o Centro de Pesquisa e Capacitação em Energia Solar da Universidade Federal de Santa Catarina (UFSC) e o Centro de Energias Renováveis da Universidade Federal de Pernambuco (UFPE).

Algumas ações nesse sentido foram propostas pela indústria nacional como a constituição do Grupo Setorial de Sistemas Fotovoltaicos pela Associação Brasileira da Indústria Elétrica e Eletrônica (ABINEE) em 2010, que resultou em a criação de itinerários e cursos formativos na área fotovoltaica dentro da parceria entre o Serviço Nacional de Aprendizagem Industrial (SENAI) e a cooperação Internacional da sociedade Alemã através do Deutsche Gesellschaft for Internacionale Zusammenarbeit (GIZ). Vale ressaltar que todas essas propostas têm por objetivo a formação de mão de obra e conta com turmas maioritariamente masculinas. Isto foi 


\section{COBENCE de Educação em Engenharia da ABENGE}

"Os desafios para formar hoje o engenheiro do amanhã"

verificado desde a formação dos docentes para ministrar as capacitações, visto que o número de professoras capacitadas não chegou nem a $10 \%$ do total.

Figura 1 - Problemas encontrados em instalações fotovoltaicas no Brasil.

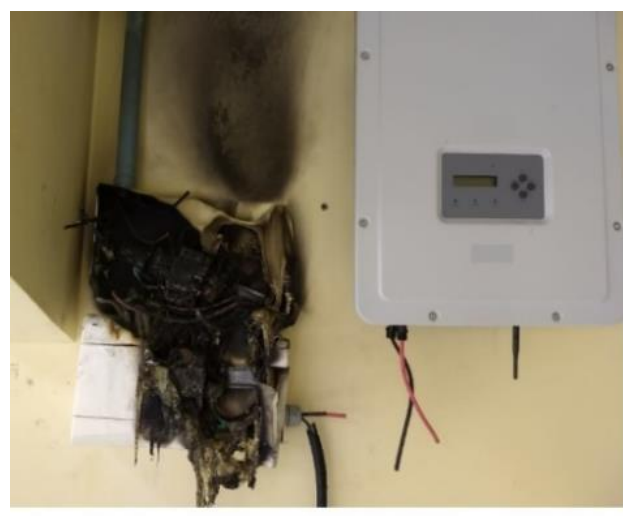

(a)

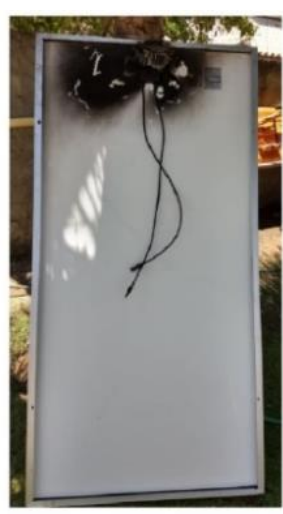

(b)

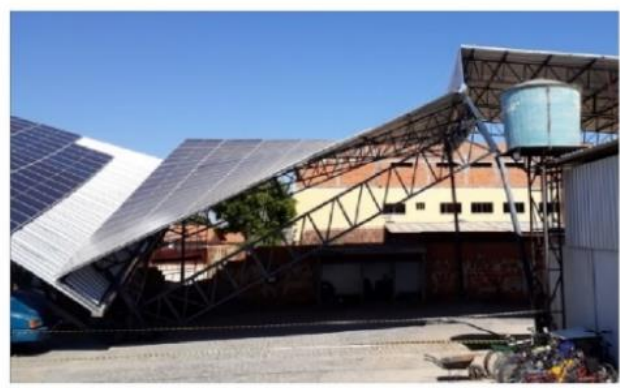

(c)

Fonte: Autor.

Desde de 2018, o Instituto Federal de Pernambuco (IFPE) Campus Pesqueira realiza capacitações semestralmente com profissionais da região para prepará-los adequadamente para o mercado de trabalho. Nas três edições concluídas desta capacitação, de um total de 71 alunos matriculados, apenas três eram do sexo feminino e dessas três, apenas duas concluíram o curso, comprovando a baixa procura das mulheres para a área.

A partir destes dados, o curso de Engenharia Elétrica do IFPE Campus Pesqueira implementou um curso de extensão de formação inicial e continuada (FIC) de 40 horas em Projetista e instaladora de sistema solar fotovoltaico, exclusivo para o publico feminino, para motivar mais mulheres a trabalhar na área da energia solar. Neste trabalho será apresentado a metodologia e a matriz curricular do curso, bem como as práticas desenvolvidas pelas alunas.

\section{CURSO DE PROJETISTA E INSTALADORA DE SISTEMAS FOTOVOLTAICOS}

A criação do curso de projetista e instaladora de sistema solar fotovoltaico no IFPE Campus Pesqueira foi possivel devido à existência do curso de técnico em eletricidade formando profissionais desde 1993 (27 anos), do curso de Bacharelado em Engenharia Elétrica fundado em 2017 e das capacitações em Sistemas Fotovoltaicos que já vem sendo ofertados na modalidade FIC desde 2018. Também foi levada em consideração para a criação do curso a existência de um grande número de egressas formadas no curso técnico em eletrotécnica da instituição com experiências práticas em instalações elétricas residenciais e industriais; de que a região do agreste do estado de Pernambuco possui um grande potencial para instalação de sistemas fotovoltaicos devido ao alto nível de irradiância solar da ordem de $5,5 \mathrm{kWh} / \mathrm{m}^{2}$ (PEDROSA FILHO, 2018); que há uma tendência de desenvolvimento de projetos de qualquer nível na região e que a formação de um grupo de mulheres capaz de instalar e projetar esse tipo de sistema permite um melhor desenvolvimento profissional no sentido de empreender seu próprio negócio na área e de motivar novas mulheres a fazer a capacitação.

O curso teve a duração de duas semanas e foi ofertado no turno da noite devido as alunas estudarem durante a manhã e a tarde. As aulas foram ofertadas de segunda a sexta com três horas de duração e aos sábados, com cinco horas de duração, totalizando 40 horas de 
formação. Os sábados foram utilizados para a realização das práticas. A Tabela 1 apresenta um resumo do conteúdo do curso. O módulo I apresenta os principais conteúdos que insere as alunas no mundo fotovoltaico, como radiação solar, células solares fotovoltaicas e módulos, o que permite que elas entendam os termos tecnológicos necessários para instalar ou projetar os sistemas. O módulo II apresentou as alunas os fundamentos básicos para projetar e Instalar sistemas fotovoltaicos a partir da elaboração do projeto do sistema fotovoltaico da sua residência, abordando os padrões brasileiros necessários para o seu desenvolvimento e o dimensionamento dos componentes da instalação. Por fim, a última etapa deste módulo abordou as práticas de instalação de sistemas conectados à rede fotovoltaica, através de tópicos relacionados à preparação, instalação e ao comissionamento desses sistemas, bem como a todos os equipamentos e padrões necessários para realizar esta atividade, finalizando com a prática de instalação de módulos fotovoltaicos em dois tipos de telhado: cerâmico e de fibrocimento.

Tabela 1 - Tópicos abordados durante a capacitação.

\begin{tabular}{|c|c|c|}
\hline Módulo & Temática & Tópicos \\
\hline 1 & $\begin{array}{c}\text { Fundamento da Energia } \\
\text { Solar Fotovoltaica }\end{array}$ & $\begin{array}{l}\text { Fundamentos da energia solar: radiação } \\
\text { solar, definições de irradiância e irradiação, } \\
\text { bancos de dados de irradiação, mapas } \\
\text { solarimétricos e instrumentação; } \\
\text { Célula e módulo fotovoltaico: efeito } \\
\text { fotovoltaico, células e módulos, características } \\
\text { elétricas e mecânicas, coeficientes de } \\
\text { temperatura. }\end{array}$ \\
\hline 2 & $\begin{array}{l}\text { Básico em projeto e } \\
\text { Instalação de sistemas } \\
\text { fotovoltaicos }\end{array}$ & $\begin{array}{l}\text { Componentes de um sistema fotovoltaico } \\
\text { conectados à rede: inversor, string box, } \\
\text { dispositivos de proteção contra sobretensão, } \\
\text { fusíveis, chave seccionadora, conectores, cabos } \\
\text { CC, estruturas mecânicas; } \\
\text { Sistemas fotovoltaicos conectados a rede: } \\
\text { Funcionamento e Dimensionamento de } \\
\text { sistemas de baixa potência; } \\
\text { Segurança nas instalações de sistemas } \\
\text { fotovoltaicos em telhado: Ferramentas e EPIs, } \\
\text { Normas de Segurança: NR-10 e NR- 35; } \\
\text { Instalação em telhados: Material de Suporte e } \\
\text { Fixação, Layout Mecânico; } \\
\text { Comissionamento de sistemas }\end{array}$ \\
\hline
\end{tabular}

Fonte: Autor.

As atividades práticas de medição e instalação foram realizadas nos sábados pela manhã já as atividades práticas sem essa finalidade forma realizadas no turno da noite. Os dois módulos do curso apresentavam atividades práticas que foram realizadas no IFPE Campus Pesqueira, por ele possuir uma usina solar fotovoltaica de $42 \mathrm{~kW}$ com módulos instalados sob diferentes condições: no telhado das salas de aula e no solo (Figura 2a), além de possuir módulos fotovoltaicos de diferentes tecnologias: monocristalinos, policristalinos, filmes finos e flexíveis e dois telhados para práticas (Figura 2b), um deles com telha cerâmica e outro com telha de fibrocimento; além disso, há também uma estação solarimétrica com feixe, medição de irradiância global e difusa (Figura 2c). 


\section{COBENCE de Educação em Engenharia

Figura - 2: (a) Arranjo fotovoltaico de $42 \mathrm{kWp}$ (b) Telhados de Práticas e (c) Estação solarimétrica.

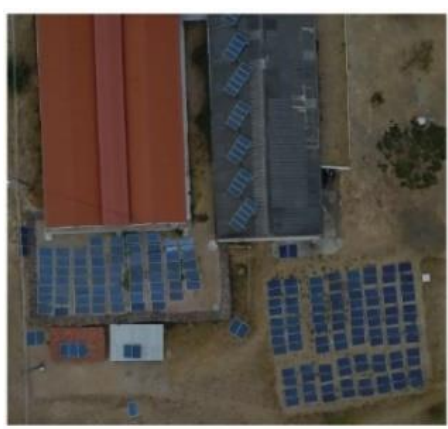

(a)

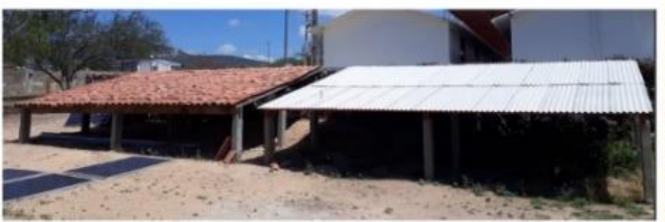

(b)

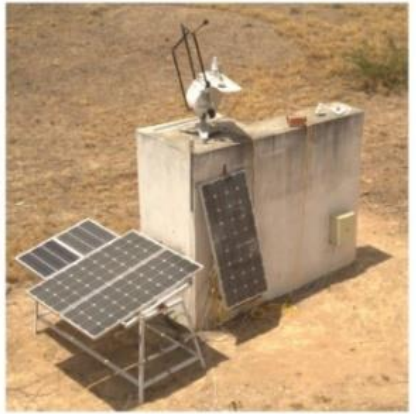

(c)

Fonte: Autor.

As práticas realizadas no primeiro Módulo do curso estão relacionadas ao procedimento de medições das grandezas elétricas do sistema fotovoltaico, práticas de conexões de módulos (serie, paralelo), mostrado na Figura 3a, montagem da String Box com diferentes configurações (Figura 3b), medições de radiação solar e busca de bancos de dados públicos de radiação solar no laboratório de informática. As práticas do Módulo II consistem na montagem dos conectores MC4 (Figura 4a), a instalação dos módulos nos telhados de teste (Figura 4b e Figura 4c), usando todos os equipamentos de proteção, e em seguida, a realização dos testes básicos de comissionamento. É importante destacar que o telhado onde são realizadas as práticas foi projetado para esta finalidade, apresentando altura máxima necessária para que não seja considerado um trabalho em altura, já que a parte mais alta dele tem apenas 1 metro de altura do solo, como pode ser vista na Figura $2 b$.

Figura - 3: Práticas realizadas no Módulo I (a) Prática de conexão de módulos e (b) Prática de montagem de String Box.

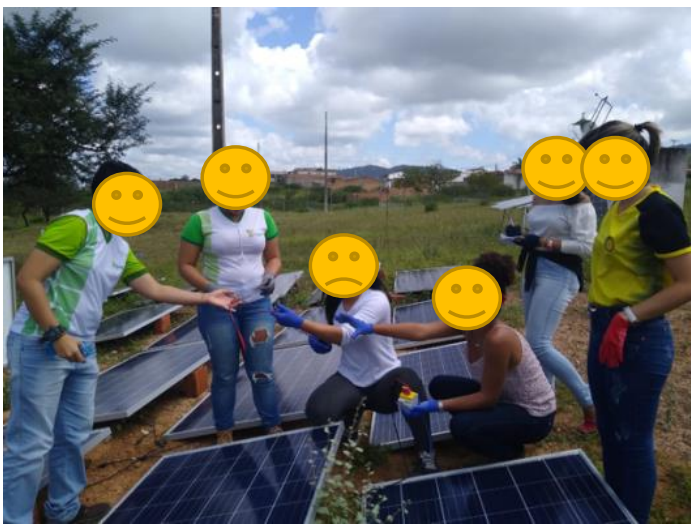

(a)

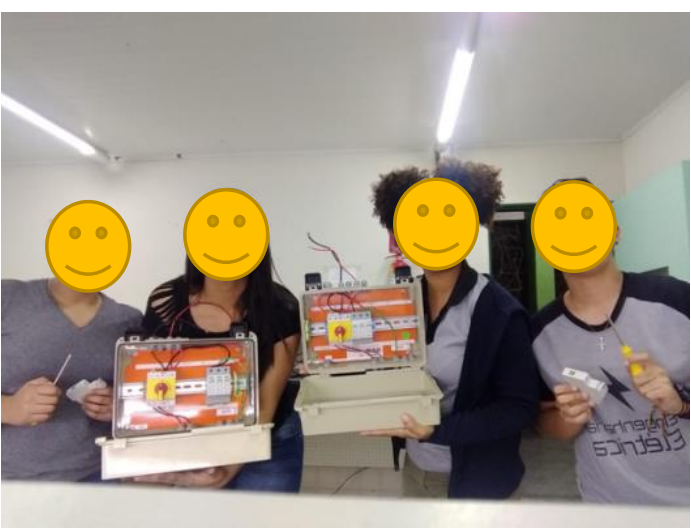

(b)

Fonte: Autor.

A Tabela 2 apresenta um resumo das atividades práticas realizadas no curso. É importante destacar que as alunas já tinham o conhecimento prévio em eletricidade básica, visto que elas já tinham cumprido os créditos da disciplina de Fundamentos da Eletricidade do curso de Bacharelado em Engenharia Elétrica. 
Figura - 4: Práticas realizadas no Módulo II (a) Instalação dos módulos, (b) Conexões elétricas dos módulos no telhado e (c) Crimpagem dos conectores MC4.

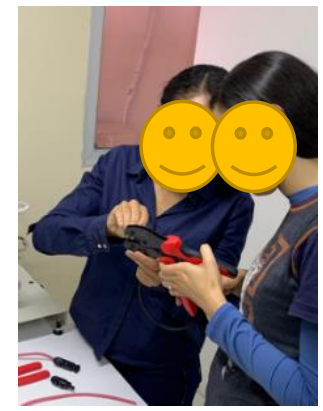

(a)

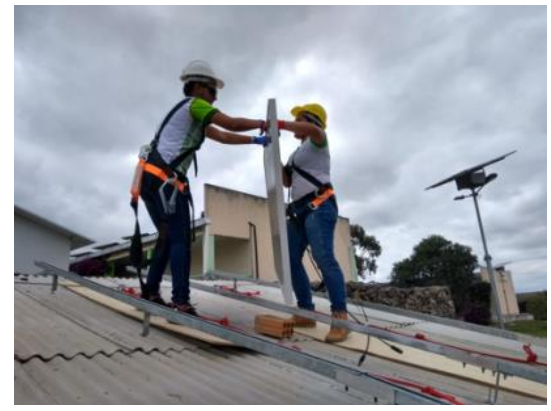

(b)

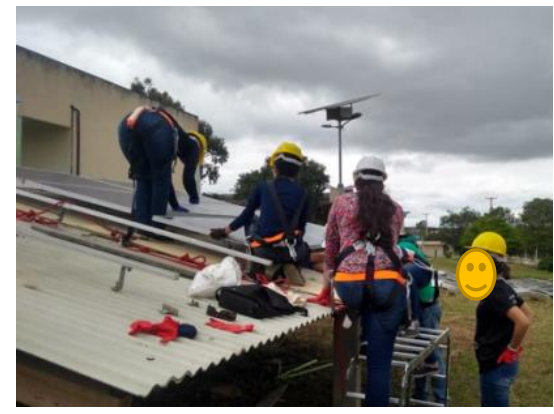

(c)

Fonte: Autor.

Tabela - 2: Detalhamento das atividades práticas desenvolvidas durante o curso.

\begin{tabular}{|c|c|c|c|c|}
\hline & Atividade & Descrição & $\begin{array}{c}\text { Duração } \\
\text { (horas) }\end{array}$ & $\begin{array}{c}\text { Conhecimento } \\
\text { necessário }\end{array}$ \\
\hline 1 & $\begin{array}{l}\text { Conexões de módulos } \\
\text { fotovoltaicos }\end{array}$ & $\begin{array}{l}\text { Abordagem das características elétricas } \\
\text { estudadas no módulo I sobre conexões } \\
\text { serie e paralelo de módulos } \\
\text { fotovoltaicos. As medições elétricas de } \\
\text { tensão e corrente são realizadas nas } \\
\text { principais configurações e diversos } \\
\text { número de módulos conectados. }\end{array}$ & 4 & $\begin{array}{l}\text { Fundamentos da } \\
\text { Eletricidade e da } \\
\text { Energia Solar } \\
\text { Fotovoltaica }\end{array}$ \\
\hline 2 & $\begin{array}{l}\text { Demonstração de softwares } \\
\text { Solarimetricos }\end{array}$ & $\begin{array}{l}\text { Demonstração da dependência } \text { da } \\
\text { radiação solar diária e mensal } \\
\text { localização, azimute e inclinação do } \\
\text { módulo, permitindo estimar seus } \\
\text { valores para situações práticas. }\end{array}$ & 1 & $\begin{array}{l}\text { Fundamentos da } \\
\text { Energia Solar } \\
\text { Fotovoltaica }\end{array}$ \\
\hline 3 & $\begin{array}{l}\text { Desenvolvimento de um } \\
\text { projeto de Sistema } \\
\text { fotovoltaico conectado à } \\
\text { rede }\end{array}$ & $\begin{array}{l}\text { Desenvolvimento de um projeto } \\
\text { completo e real de um cliente } \\
\text { residencial, utilizando todo o } \\
\text { conhecimento construído sobre energia } \\
\text { solar fotovoltaica, rede e padrões. } \\
\text { Alguns detalhes do projeto são } \\
\text { apresentados, como: materiais e listas } \\
\text { de preços, desenhos dos detalhes da } \\
\text { instalação e documentação do projeto } \\
\text { necessária para que possa ser aprovada } \\
\text { pelas concessionárias de energia. }\end{array}$ & 6 & $\begin{array}{l}\text { Fundamentos da } \\
\text { Eletricidade, da } \\
\text { Energia Solar } \\
\text { Fotovoltaica e } \\
\text { de projetos de } \\
\text { Sistemas } \\
\text { Fotovoltaicos. }\end{array}$ \\
\hline 4 & \begin{tabular}{llr} 
Projeto e & \multicolumn{2}{c}{ Instalação da } \\
proteção de & sistemas \\
Fotovoltaicos
\end{tabular} & $\begin{array}{l}\text { Montagem de diversas configurações } \\
\text { de entrada e saída de String Box e } \\
\text { conectores MC4 }\end{array}$ & 3 & $\begin{array}{l}\text { Fundamento de } \\
\text { energia Solar e } \\
\text { dos } \\
\text { componentes de } \\
\text { um sistema }\end{array}$ \\
\hline 5 & $\begin{array}{l}\text { Instalação de sistemas } \\
\text { fotovoltaicos em telhado }\end{array}$ & 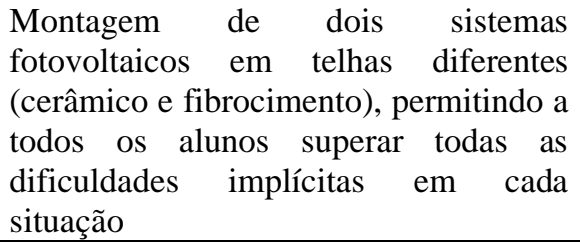 & 5 & $\begin{array}{l}\text { Todo o conteúdo } \\
\text { abordado no } \\
\text { curso. }\end{array}$ \\
\hline 6 & Comissionamento & $\begin{array}{l}\text { A execução dos procedimentos de } \\
\text { segurança necessários para realizar a } \\
\text { conexão à rede. }\end{array}$ & 1 & $\begin{array}{l}\text { Todo o conteúdo } \\
\text { abordado no } \\
\text { curso. }\end{array}$ \\
\hline
\end{tabular}

Fonte: Autor. 


\section{CONSIDERAÇÕES FINAIS}

A Primeira edição do curso de capacitação em sistemas fotovoltaicos para mulheres foi realizada no segundo semestre de 2019 durante o mês de agosto. A escolha das datas foi definida para que não houvesse choques com as atividades acadêmicas das alunas. A turma foi composta de onze estudantes do curso de bacharelado em Engenharia Elétrica e uma docente do curso (Figura 5). Ao final do curso as alunas relataram que o curso foi bem intenso, porém muito proveitoso. $\mathrm{O}$ fato de o curso ter sido realizado no terceiro turno de aulas, fez com que as alunas ao final da semana chegassem cansadas, porém não houve desmotivação e as atividades foram concluídas com êxito. Foi observado que as alunas foram capazes de realizar as mesmas atividades que propomos as capacitações com o grupo majoritariamente masculino. A Figura 6 comprova esta afirmação, demostrando que nem a realização de atividades que exigiam força foi capaz de desmotiva-las.

Figura - 5: Alunas concluintes da primeira capacitação em Sistemas Fotovoltaicos para mulheres.

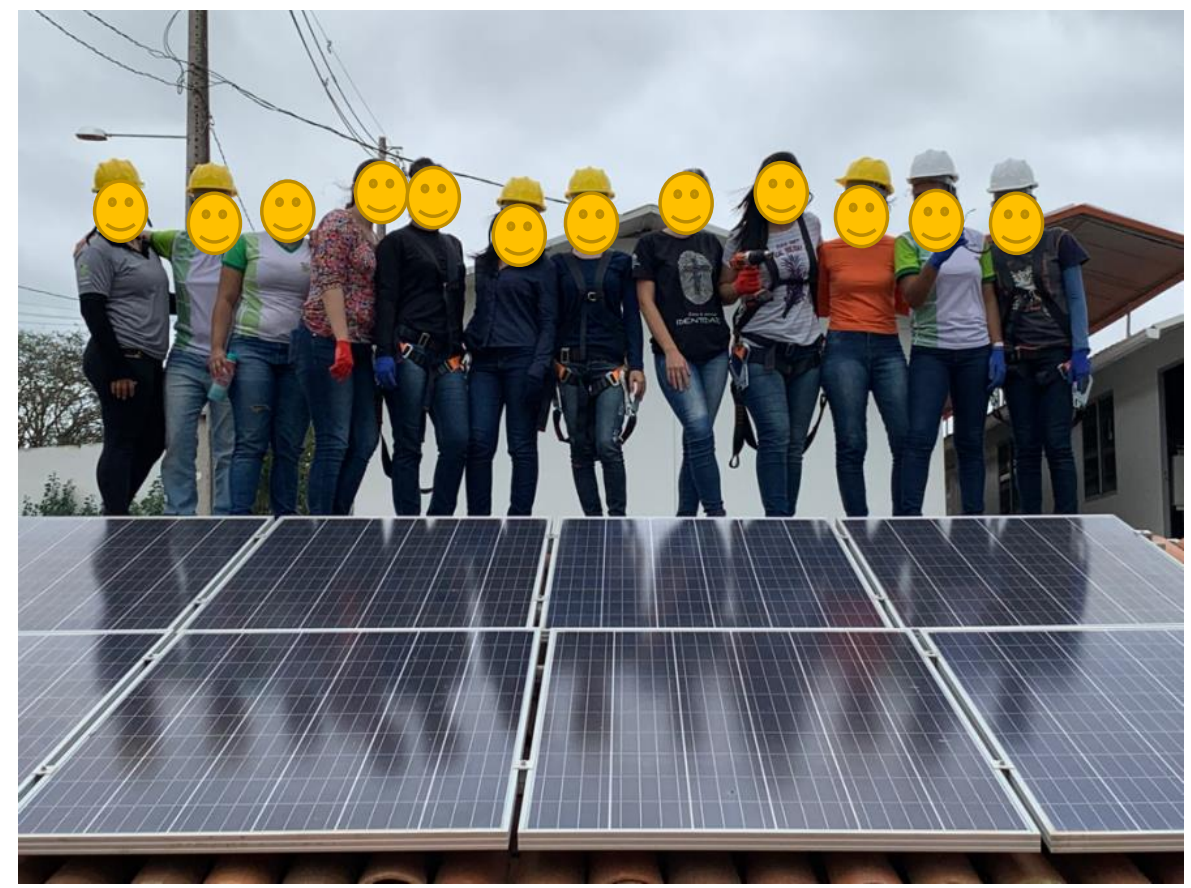

Fonte: Autor.

Espera-se realizar esta capacitação novamente com as novas turmas de engenharia e motivar as alunas e docente formadas na primeira turma a auxiliar nas atividades da nova turma, para que as novas alunas se sintam ainda mais motivada por estarem sendo treinadas por mulheres. Como resultado desta capacitação, observou-se o engajamento de algumas alunas em projetos de pesquisa na área da energia solar fotovoltaica bem como uma das alunas iniciou atividades de estágio em uma empresa de projetos e instalação de sistemas fotovoltaicos da cidade, formado por ex-alunos das capacitações ofertadas anteriormente. 


\section{COBENCE

Figura - 6: Instalação dos módulos feito pelas alunas.

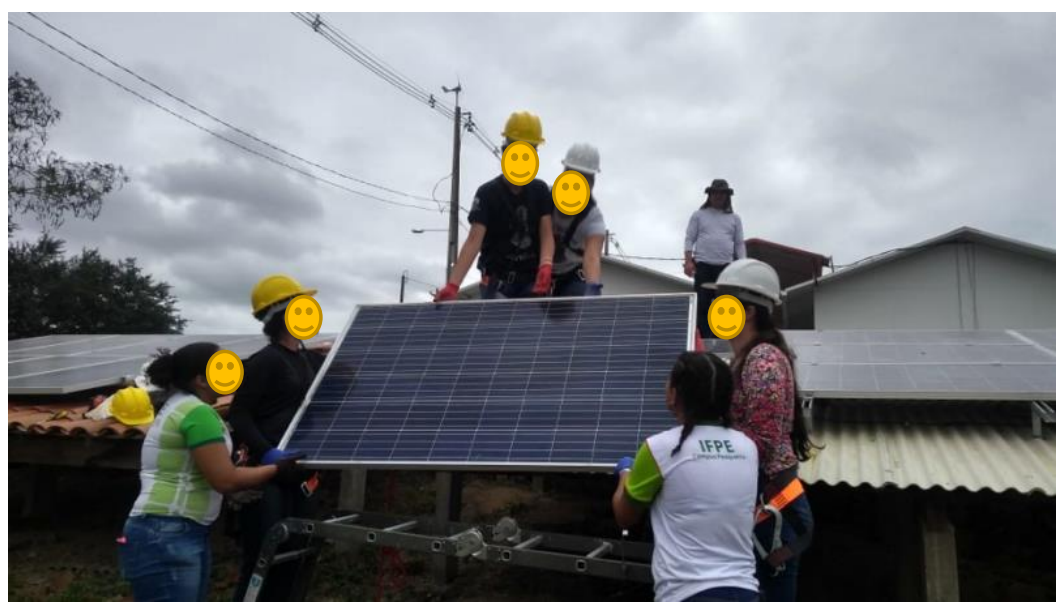

Fonte: Autor.

\section{AGRADECIMENTOS}

Agradecemos ao IFPE Campus Pesqueira e as empresas parceiras em cooperação técnica pelo suporte aos cursos ministrados.

\section{REFERÊNCIAS}

BLASQUES, L. A educação profissional e tecnológica e os itinerários formativos como potenciais soluções para a escassez de mão de obra especializada em instalações FV no Brasil. In: VI Congresso Brasileiro de Energia Solar, 2016, Minas Gerais. Anais. Belo Horizonte, 2016.

DINIZ, M.M.N. et al. Experiência do Instituto Juazeiro na Promoção de Cursos Técnicos na Área de Sistemas Fotovoltaicos. In: VI Congresso Brasileiro de Energia Solar, 2016, Minas Gerais. Anais. Belo Horizonte, 2016.

MOCELIN, André Ricardo. Qualificação profissional e capacitação laboratorial em sistemas fotovoltaicos. 2014. 200 f. Tese (Doutorado) - Instituto de Energia e Ambiente, Universidade de São Paulo, São Paulo, 2014.

PEDROSA FILHO, M.H.O. et al. Desenvolvimento de itinerários formativos para a educação profissional e tecnológica na área de energia solar fotovoltaica. In: VII Congresso Brasileiro de Energia Solar, 2018, Porto Alegre. Anais. Gramado, 2018.

PEDROSA FILHO, M.H.O., Mariano, V., 2018. Análise da tendência da irradiação solar e temperatura ambiente no agreste nordestino empregando dados medidos de longo prazo. In: VII Congresso Brasileiro de Energia Solar, 2018, Porto Alegre. Anais. Gramado, 2018. 


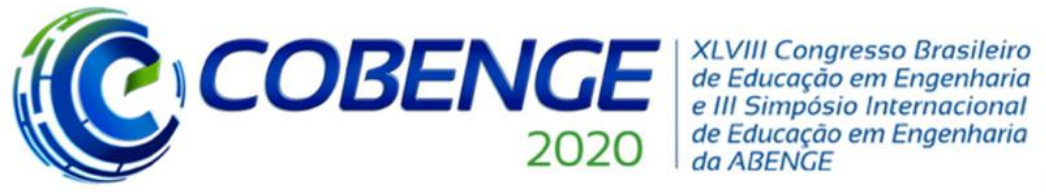

"Os desafios para formar hoje o engenheiro do amanhã"
$\mathrm{O} 1$ a $\mathrm{O} 3$ de dezembro

\section{Evento On-line}

\title{
WOMEN IN ENGINEERING: SOLAR ENERGY TRAINING FOR GIRLS.
}

\begin{abstract}
With the publication of Normative Resolution 482/12 and 687/15 by ANEEL, the generation of photovoltaic solar energy in Brazil began to achieve exponential growth, providing the emergence of the demands of professionals to work in projects and systems installations. From this moment on, several short courses began to be offered with the objective of remedying this deficiency. However, it was observed that these qualifications were not sufficient to qualify a professional in this area and from then on, a discussion started between the main technological education institutions to create a formative itinerary that would enable these professionals to be trained more proper. This work presents the methodology and results of a training model carried out specifically for a group of students and teachers from the Federal Institute of Pernambuco in projects and installation of photovoltaic systems, with the aim of motivating them to explore areas that historically only men did and the results were also used to motivate new students to work in this area. This work will also describe the development of contents addressed to the training of students to meet the needs of the market, such as concepts, techniques and basic materials of photovoltaic solar energy for roof installations. The main practices carried out are also described, such as the assembly of the components of the MC4 connector, of the String Box and the installation procedures of the photovoltaic modules on the roof (safety and materials).
\end{abstract}

Keywords: Education in photovoltaics systems, Women in Engineering, Grid connected solar systems. 